\title{
Expectation of a uniform random variable with uniform observation errors after selection of the highest observations
}

\author{
Cornelis A. Van Bochove
}

Received: 2 March 2009 / Revised: 17 December 2009 / Published online: 14 January 2010

(C) The Author(s) 2010. This article is published with open access at Springerlink.com

\begin{abstract}
In selection processes of a random variable with random observation errors, the crucial variable is the conditional expectation of the target variable given the sum of the observations. An example is the selection of the most talented young researchers for tenure track. This paper derives an explicit expression for this conditional expectation for the case that both the target variable and the observation errors have a uniform, but different, distribution.
\end{abstract}

Keywords Uniform distribution - Selection - Conditional expectation · Observation error $\cdot$ Research career

Mathematics Subject Classification (2000) $\quad 62 \mathrm{E} 15$

\section{Introduction}

This paper derives the conditional expectation of a random variable with a uniform distribution, given the sum of this variable and of an arbitrary number of independent variables with a second uniform distribution. The need to obtain this conditional expectation arises in the computation of the expected value of a uniform random variable with independent uniform observation errors, given the sum of a number of observations. The problem was inspired by an analysis of the selection of the most talented young researchers for tenure track. Research talent (the target variable) cannot be observed directly, but has to be inferred from actual research output over a number of years. An individual's output can be considered as the sum of his or her research talent

C. A. Van Bochove $(\varangle)$

Centre for Science and Technology Studies (CWTS), Leiden University, Leiden, The Netherlands

e-mail: cbochove@cwts.nl

URL: http://www.socialsciences.leidenuniv.nl/cwts/ 
(appropriately defined) and an annual random component (the 'observation error'). If the $s$ percent researchers with the highest observed output are selected for tenure track, the expected value of the target variable within this group increases with the length of the selection period. This increased accuracy has to be balanced against the cost of extending the selection period. This requires the computation of the expected value of the target variable in the upper $s$ percent of the distribution of actual output after a number of years, which is equivalent to obtaining the conditional expectation of the target variable given the sum of the observations over the selection period.

For some purposes ${ }^{1}$ a numerical computation of the conditional expectation is insufficient and an analytical expression is needed. Obtaining it requires an analytical expression for the distribution function of the sum of the observations. For most distributions this is not available. However, if both the target variable and the observation errors have uniform distributions, an analytical expression of the distribution of the sum is a special case of a result recently published in Statistical Papers (Sadooghi-Alvandi et al. 2009). We will show that in this case it also possible to derive an analytical expression for the conditional expectation. ${ }^{2}$

\section{Implicit expression for the conditional expectation}

Formally, we consider the case of a random variable $V$ with a uniform distribution on $(0, b)$. Let there be $n-1$ observations of $V$, each having a random error with another uniform distribution. We will denote the error of observation $i$ by $X_{i+1}$. This allows us to define the sum $S$ of $n-1$ observations as $S=\sum_{i=1}^{n} X_{i}$, where $X_{1}=(n-1) V$. Then $S$ is the sum of $n$ independent random variables $X_{i}$, with $X_{1}$ uniform on $\left(0, a_{1}\right), a_{1}=$ $(n-1) b$, and $X_{i}(i \geq 2)$ uniform on $\left(0, a_{2}\right)$; consequently $0 \leq S \leq a_{1}+(n-1) a_{2}$. We are interested in the expectation of $X_{1}$ given the value of $S: E\left(X_{1} \mid S>s\right)=$ $\int_{0}^{a_{1}} x_{1} f_{X_{1} \mid S}\left(x_{1} \mid s\right) \mathrm{d} x_{1}$, where $f_{X_{1} \mid S}$ is the conditional probability density function (pdf) of $X_{1}$ given $S>s$. The corresponding conditional distribution function is:

$$
\begin{aligned}
F_{X_{1} \mid S}\left(x_{1} \mid S\right) & =P\left[X_{1} \leq x_{1} \mid S>s\right]=\frac{P\left[X_{1} \leq x_{1} \wedge S>s\right]}{P[S>s]} \\
& =\frac{P\left[X_{1} \leq x_{1}\right]-P\left[X_{1} \leq x_{1} \wedge S<s\right]}{1-P[S<s]} \\
& =\frac{F_{X_{1}}\left(x_{1}\right)-P\left[X_{1} \leq x_{1} \wedge S_{2}+X_{1}<s\right]}{1-F(s)}
\end{aligned}
$$

where $S_{2}=\sum_{i=2}^{n} X_{i}$ is the sum of the observation errors, $F_{X_{1}}\left(x_{1}\right)$ is the marginal distribution of $x_{1}$ and $F(s)$ is the distribution function of $S$. For $x_{1}>s$, the probability in the extreme right hand side of (1) is:

\footnotetext{
${ }^{1}$ In our case this purpose was the development of a simulation model of tenure track policy in a national research system. Such a model requires a specification of the relation between average research productivity, and the rate and speed of selection.

2 Though this work has been inspired by the specific case of the design of tenure track systems, it is also applicable to the design of other selection processes, provided uniform distributions are at least a reasonable first approximation for the target variable and the observation errors.
} 


$$
\begin{aligned}
P\left[X_{1} \leq x_{1} \wedge S_{2}+X_{1}<s\right]= & P\left[s<X_{1} \leq x_{1} \wedge S_{2}+X_{1}<s\right] \\
& +P\left[X_{1}<s \wedge S_{2}+X_{1}<s\right] \\
= & P\left[X_{1}<s \wedge S_{2}+X_{1}<s\right]
\end{aligned}
$$

where the last equality follows from the fact that the first of the two probabilities in the second expression is zero if $x_{1}>s$. Defining $f_{2}\left(s_{2}\right)$ as the pdf of $S_{2}$, the independence of $X_{1}$ and $S_{2}$ implies:

$$
P\left[X_{1}<x_{1} \wedge S_{2}+X_{1}<s\right]=\int_{0}^{\min \left\{x_{1}, s\right\}} \int_{0}^{s-y_{1}} f_{X_{1}}\left(y_{1}\right) f_{2}\left(y_{2}\right) \mathrm{d} y_{2} \mathrm{~d} y_{1}
$$

The conditional pdf is now given by:

$$
\begin{aligned}
f_{X_{1} \mid S}\left(x_{1} \mid s\right)= & \frac{d F_{X_{1} \mid S}\left(x_{1} \mid s\right)}{d x_{1}}=\frac{1}{1-F(s)} \\
& \times\left\{f_{X_{1}}\left(x_{1}\right)-\frac{\mathrm{d} \int_{0}^{\min \left\{x_{1}, s\right\}} \int_{0}^{s-y_{1}} f x_{1}\left(y_{1}\right) f_{2}\left(y_{2}\right) \mathrm{d} y_{2} \mathrm{~d} y_{1}}{\mathrm{~d} x_{1}}\right\}
\end{aligned}
$$

Boundary conditions of the second integral in (4) stem from $f_{2}\left(y_{2}\right)=0$ for $y_{2}<0$ and for $y_{2}>(n-1) a_{2}$. For notational compactness it is convenient to define $[x]_{+}=$ $\max (x, 0) ;[x]_{+}^{i}=[\max (x, 0)]^{i}$; the Dirac operator $\delta(z)=0,1 \Leftrightarrow z \leq 0,>0$; and the function $z_{x}(i)=x-i a_{2}$. The integral in (3) and (4) can now be written as:

$$
\begin{aligned}
& \delta\left(z_{s}(n-1)\right) \int_{0}^{\min \left\{x_{1}, z_{s}(n-1)\right\}} f_{X_{1}}\left(y_{1}\right) \mathrm{d} y_{1} \\
& +\delta\left(x_{1}-z_{s}(n-1)\right) \int_{\left[z_{s}(n-1)\right]_{+}}^{\min \left\{x_{1}, s\right\}} f_{X_{1}}\left(y_{1}\right) F_{2}\left(s-y_{1}\right) \mathrm{d} y_{1}
\end{aligned}
$$

Evaluation of the integrals as far as possible and substitution of $f_{X_{1}}\left(x_{1}\right)=\frac{1}{a_{1}}$ yields:

$$
\begin{aligned}
& \int_{0}^{\min \left\{x_{1}, s\right\}} \int_{0}^{s-y_{1}} f_{X_{1}}\left(y_{1}\right) f_{2}\left(y_{2}\right) \mathrm{d} y_{2} \mathrm{~d} y_{1}=\delta\left(z_{s}(n-1)\right) \frac{\min \left\{x_{1}, z_{s}(n-1)\right\}}{a_{1}} \\
& \quad+\delta\left(x_{1}-z_{s}(n-1)\right) \int_{\left[z_{s}(n-1)\right]_{+}}^{\min \left\{x_{1}, s\right\}} \frac{F_{2}\left(s-y_{1}\right)}{a_{1}} \mathrm{~d} y_{1}
\end{aligned}
$$


Differentiating the right hand side of (6) with respect to $x_{1}$ and inserting the result in (4) we find:

$$
f_{X_{1} \mid S}\left(x_{1} \mid s\right)=\delta\left(x_{1}-z_{s}(n-1)\right) \frac{1-\delta\left(s-x_{1}\right) F_{2}\left(s-x_{1}\right)}{a_{1}(1-F(s))}
$$

From which the conditional expectation is seen to be:

$$
E\left(X_{1} \mid S>s\right)=\frac{a_{1}^{2}-\left[z_{s}(n-1)\right]_{+}^{2}}{2 a_{1}(1-F(s))}-\int_{\left[z_{s}(n-1)\right]_{+}}^{\min \left\{s, a_{1}\right\}}\left\{\frac{x_{1} F_{2}\left(s-x_{1}\right)}{a_{1}(1-F(s))}\right\} \mathrm{d} x_{1}
$$

\section{Explicit expression for the conditional expectation}

Sadooghi-Alvandi et al. (2009) provide a general expression for the pdf of the sum $S$ of $n$ independent uniform variables $X_{i}$ with $0<X_{i}<b_{i}$ :

$$
f(s)=\frac{1}{(n-1) ! \Pi_{1}^{n} b_{i}}\left\{s^{n-1}+\sum_{k=1}^{n}(-1)^{k} \sum_{J_{k}}\left[s-\sum_{l=1}^{k} b_{j_{l}}\right]_{+}^{n-1}\right\},
$$

where $J_{k}=\left\{\left(j_{1}, j_{2}, \ldots, j_{k}\right) ; 1 \leq j_{1}<j_{2}<\cdots<j_{k} \leq n\right\}$, the $j_{i}$ 's being integer. In our case the $X_{i}$ are identical for $i \geq 2$, implying $b_{j_{l}}=a_{2}$ for $j_{l}>1$. There are $\left(\begin{array}{c}n-1 \\ k-1\end{array}\right)$ k-tuples with $j_{1}=1$ and $\left(\begin{array}{c}n-1 \\ k\end{array}\right)$ k-tuples with all $j_{l}$ 's $>1$. Let $\delta_{n k}$ denote the Kronecker delta. The density function of S now is:

$$
\begin{aligned}
f(s)= & \frac{1}{a_{1} a_{2}^{n-1}(n-1) !} \sum_{k=0}^{n}(-1)^{k}\left\{\left(\begin{array}{c}
n-1 \\
k-1
\end{array}\right)\left(1-\delta_{k 0}\right)\left[z_{s}(k-1)-a_{1}\right]_{+}^{n-1}\right. \\
& \left.+\left(1-\delta_{n k}\right)\left(\begin{array}{c}
n-1 \\
k
\end{array}\right)\left[z_{s}(k)\right]_{+}^{n-1}\right\}
\end{aligned}
$$

The conditional expectation in (8) contains both the distribution of $S, F(s)$, and the distribution of the sum of the observation errors, $F_{2}\left(s_{2}\right)$. To obtain the pdf of the sum of the observation errors, $f_{2}\left(s_{2}\right)$, from (9), we first set $a_{1}=a_{2}$ :

$$
f(s)=\frac{1}{a_{2}^{n}(n-1) !} \sum_{k=0}^{n}(-1)^{k}\left(\begin{array}{l}
n \\
k
\end{array}\right)\left[z_{s}(k)\right]_{+}^{n-1}
$$

and next substitute $n-1$ for $n$ :

$$
f_{2}\left(s_{2}\right)=\frac{1}{a_{2}^{n-1}(n-2) !} \sum_{k=0}^{n-1}(-1)^{k}\left(\begin{array}{c}
n-1 \\
k
\end{array}\right)\left[z_{s_{2}}(k)\right]_{+}^{n-2}
$$


To derive $F(s)$ and $F_{2}\left(s_{2}\right)$ from (9) and (10), respectively, note that

$$
\int_{0}^{s}\left[z_{y}(i)\right]_{+}^{n} \mathrm{~d} y=\delta\left(z_{s}(i)\right) \int_{-z_{0}(i)}^{s}\left(z_{y}(i)\right)^{n} \mathrm{~d} y=\frac{\left[z_{s}(i)\right]_{+}^{n+1}}{n+1}
$$

Consequently:

$$
\begin{aligned}
F(s)= & \int_{0}^{s} f(y) \mathrm{d} y=\frac{1}{n ! a_{1} a_{2}^{n-1}} \sum_{k=0}^{n}(-1)^{k}\left\{\left(1-\delta_{n k}\right)\left(\begin{array}{c}
n-1 \\
k
\end{array}\right)\left[z_{s}(k)\right]_{+}^{n}\right. \\
& \left.+\left(1-\delta_{k 0}\right)\left(\begin{array}{c}
n-1 \\
k-1
\end{array}\right)\left[z_{s}(k-1)-a_{1}\right]_{+}^{n}\right\} \\
F_{2}\left(s_{2}\right)= & \delta\left(s_{2}\right) \frac{1}{(n-1) ! a_{2}^{n-1}} \sum_{k=0}^{n-1}(-1)^{k}\left(\begin{array}{c}
n-1 \\
k
\end{array}\right)\left[z_{s_{2}}(k)\right]_{+}^{n-1}
\end{aligned}
$$

Inserting (12) and (13), the core of the integral in (8) is $(p>q>0)$ :

$$
\int_{q}^{p} x_{1} F_{2}\left(s-x_{1}\right) \mathrm{d} x_{1}=\int_{q}^{p} \frac{x_{1}}{(n-1) ! a_{2}^{n-1}} \sum_{k=0}^{n-1}(-1)^{k}\left(\begin{array}{c}
n-1 \\
k
\end{array}\right)\left[z_{s}(k)-a_{1}\right]_{+}^{n-1} \mathrm{~d} x_{1}
$$

The right hand side of (14) contains integrals of the type $\int_{q}^{p} y(c-y)_{+}^{n} \mathrm{~d} y$, where integration yields:

$$
\int_{q}^{p} y(c-y)_{+}^{n-1} \mathrm{~d} y=\frac{q(c-q)_{+}^{n}}{n}+\frac{(c-q)_{+}^{n+1}}{n(n+1)}-\frac{p(c-p)_{+}^{n}}{n}-\frac{(c-p)_{+}^{n+1}}{n(n+1)}
$$

implying:

$$
\begin{aligned}
\int_{q}^{p} x_{1} F_{2}\left(s-x_{1}\right) \mathrm{d} x_{1}= & \frac{1}{n ! a_{2}^{n-1}} \sum_{k=0}^{n-1}(-1)^{k}\left(\begin{array}{c}
n-1 \\
k
\end{array}\right)\left[q\left[z_{s}(k)-q\right]_{+}^{n}\right. \\
& \left.+\frac{\left[z_{s}(k)-q\right]_{+}^{n+1}}{n+1}-p\left[z_{s}(k)-p\right]_{+}^{n}-\frac{\left[z_{s}(k)-p\right]_{+}^{n+1}}{n+1}\right]
\end{aligned}
$$


We now obtain the desired explicit expression for the conditional expectation:

$$
\begin{aligned}
E\left(X_{1} \mid S>s\right)= & \frac{a_{1}^{2}-\left[z_{s}(n-1)\right]_{+}^{2}}{2 a_{1}(1-F(s))}-\frac{1}{a_{1} a_{2}^{n-1}(1-F(s)) n !} \sum_{k=0}^{n-1}(-1)^{k}\left(\begin{array}{c}
n-1 \\
k
\end{array}\right) \\
& \times\left\{\left[z_{s}(n-1)\right]_{+}\left[z_{s}(k)-\left[z_{s}(n-1)\right]_{+}\right]_{+}^{n}\right. \\
& +\frac{\left[z_{s}(k)-\left[z_{s}(n-1)\right]_{+}\right]_{+}^{n+1}}{n+1}-a_{1}\left[z_{s}(k)-a_{1}\right]_{+}^{n} \\
& \left.-\frac{\left[z_{s}(k)-a_{1}\right]_{+}^{n+1}}{n+1}\right\}
\end{aligned}
$$

\section{Illustration: expected error equal to expected target value, best half selected}

To illustrate the values of $E\left(X_{1} \mid S>s\right)$ as $n$ increases, we consider the case $a_{1}=$ $(n-1) a_{2}$, implying that in every period the variances (and the expected values) of the

Table 1 Expected value of the target variable in the upper half of the observations, as fraction of the upper boundary of the target variable, variances of observation errors and target variable equal

\begin{tabular}{ll}
\hline$n-1$ & $\frac{E\left(X_{1} \mid S>s\right)}{a_{1}}$ \\
\hline 1 & 0.667 \\
2 & 0.708 \\
3 & 0.722 \\
4 & 0.729 \\
5 & 0.733 \\
6 & 0.736 \\
7 & 0.738 \\
8 & 0.740 \\
9 & 0.741 \\
10 & 0.742 \\
11 & 0.742 \\
12 & 0.743 \\
13 & 0.744 \\
14 & 0.744 \\
15 & 0.744 \\
16 & 0.745 \\
17 & 0.745 \\
18 & 0.745 \\
19 & 0.746 \\
20 & 0.746 \\
\hline
\end{tabular}


observation error and the target variable are equal. We specifically consider $s=a_{1}$, implying that the conditional expectation gives the expected value of $x_{1}$ in the upper half of the actual observations. In a selection process this would mean that the best half is selected. In this case, (16) simplifies to:

$$
E\left(X_{1} \mid S>s\right)=a_{1}-\frac{2 a_{1}(n-1)^{n-1}}{(n+1) !} \sum_{k=0}^{n-1}(-1)^{k}\left(\begin{array}{c}
n-1 \\
k
\end{array}\right)\left(\frac{n-k-1}{n-1}\right)^{n+1}
$$

The value of $E\left(X_{1} \mid S>s\right)$ as a fraction of $a_{1}$ is given in Table 1. Note that this fraction converges very rapidly to 0.75 , the expected value in the upper half of the distribution if there are no observation errors. This rapid convergence occurs even though we consider the case where the variance of the observation errors is relatively large: equal to that of the target variable.

Though this finding admittedly depends to some degree on the assumption of uniform distributions, it is likely that it has important implications for the case of tenure track selection that inspired this paper. In the case of tenure track, the selection period in many countries has become very long, often 15 years or more. This is in stark contrast to the employment conditions in other sectors of society, where selection periods of a few years before appointment to a permanent post are common. Our result, obtained through elementary but rigorous statistical reasoning, indicates that it is unlikely that the far longer selection periods in the research system lead to a substantially greater accuracy in the selection of the most talented researchers.

Acknowledgements I would like to thank Ludo Waltman of CWTS and an anonymous referee for comments that improved the presentation.

Open Access This article is distributed under the terms of the Creative Commons Attribution Noncommercial License which permits any noncommercial use, distribution, and reproduction in any medium, provided the original author(s) and source are credited.

\section{Reference}

Sadooghi-Alvandi SM, Nematollahi AR, Habibi R (2009) On the distribution of the sum of independent uniform random variables. Stat Pap 50:171-175 